\title{
Operational aspects of the implementation of multidrug therapy at ALERT, Ethiopia
}

\author{
MARIJKE BECX-BLEUMINK \\ All Africa Leprosy and Rehabilitation Training Centre, P.O. Box \\ 165, Addis Ababa, Ethiopia
}

\section{Introduction}

The ALERT Leprosy Control Department is responsible for leprosy control in Shoa Administrative Region (Figure 1). This region is centrally located in Ethiopia; it covers an area of about $85,000 \mathrm{sq} \mathrm{km}$, with a population of 8.75 million. The region is divided into one urban and 11 rural districts. Leprosy diagnostic and treatment services are given in 292 centres, $176(60 \%)$ are attached to the general medical services and $116(40 \%)$ are leprosy clinics, which have been established in those areas where a general medical service does not exist yet. About $50 \%$ of the centres are accessible by car during the whole year; $32 \%$ are accessible by car during the dry season only, while $18 \%$ are not accessible by car. ${ }^{1}$

\section{Multidrug therapy (MDT) in the ALERT Leprosy Control Programme}

Multidrug therapy (MDT) according to the recommendations given by the World Health Organization (WHO) in 1982, ${ }^{2}$ was introduced in the ALERT Leprosy Control Programme in January 1983. Paucibacillary (PB) patients are treated for a period of 6 months, with dapsone daily self-administered and rifampicin monthly under supervision. Multibacillary (MB) patients are treated for a period of at least two years, and until the skin smears (Bacteriological Index) have become negative, with daily dapsone and clofazimine self-administered, and monthly rif ampicin and clofazimine under supervision. ${ }^{3}$

During 1983 MDT was introduced into two districts, Tegulet \& Bulga and Yifat \& Timuga, in the north-eastern part of the region, the Debre Berhan area, including 64 clinics. During 1984 MDT was extended to three districts, Addis Ababa, Menagesha and Yerer \& Kereyu, in the central part of the region, the Addis Ababa area, including 48 clinics.

In December 1985 MDT was extended to two further districts, Haykoch and Butajira and Kembata \& Hadiya, in the southern part of the region, the Southern 


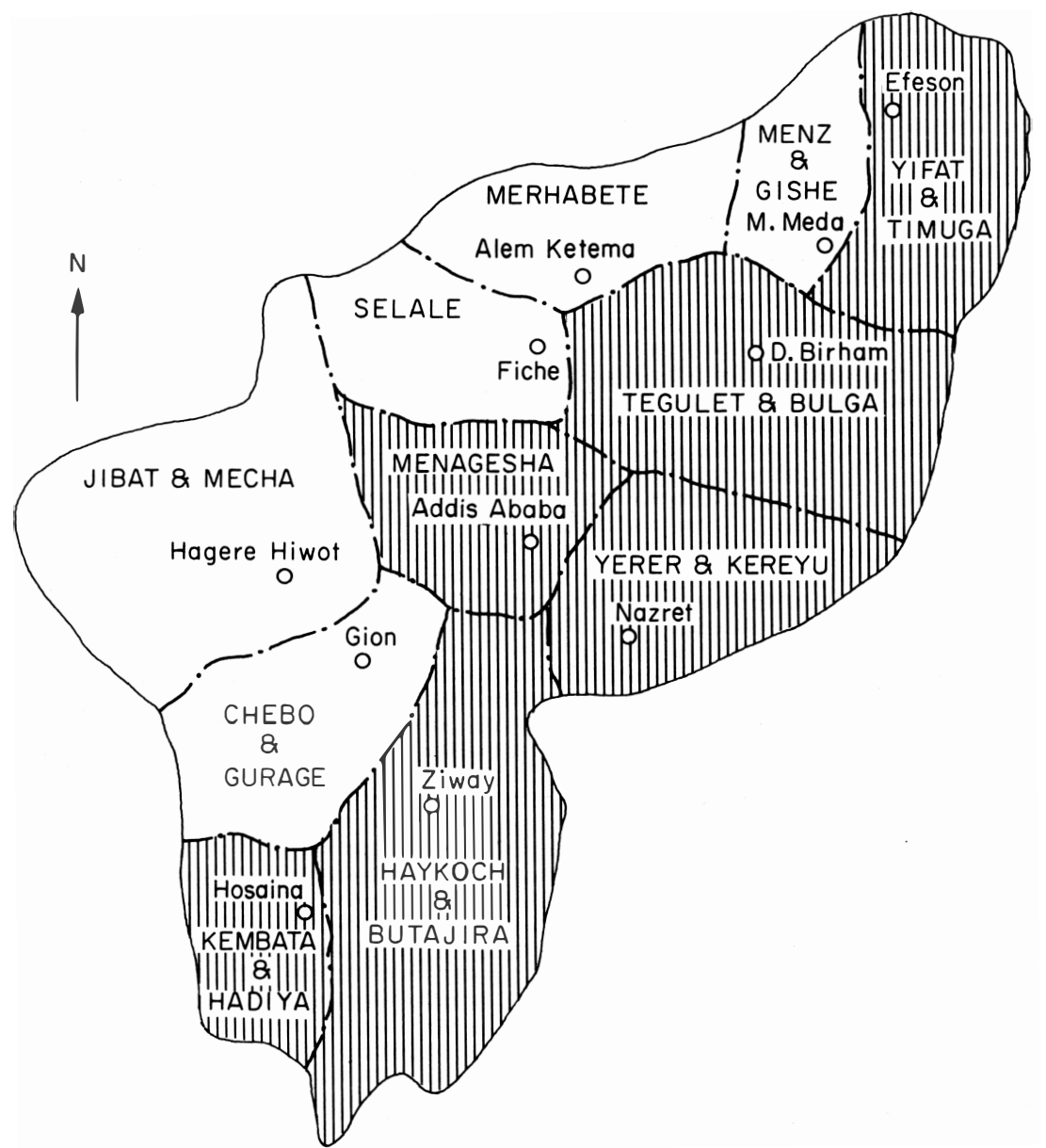

Figure 1. , administrative region boundary; Awraja capital; //, MDT area.

, Awraja boundary; $\bullet$, capital city; $\bigcirc$,

Shoa area, including 61 clinics. During the last quarter of 1986 two more districts, Selale and Menz \& Gishe, will be included in the MDT programme, while the last three districts of the region, Merhabete, Gibat \& Mecha and Chebo \& Gurage, will be included in the MDT programme during the period 1987-1989 (Figure 1).

Prior to the introduction of MDT the leprosy control services are reorganized and intensified. This includes clinical and bacteriological examination of the patients under treatment, (re)assessment of the classification, and release from treatment of those patients who are considered to have received sufficient chemotherapy with dapsone monotherapy. Furthermore, new recording and reporting systems are introduced, health education is given to patients and the communities, and the tasks and training of all cadres of staff are re-defined.

By January 1986, 7587 patients (3320 PB and $4267 \mathrm{MB}$ ) had been put under MDT. 


\section{Release from treatment prior to introduction of MDT}

During the period January 1983-January 19866005 patients (4186 PB, 1769 MB and 50 unclassified borderline) have been released from dapsone monotherapy. These patients fulfilled the criteria of regular attendance of at least 5 years for PB patients and at least 10 years for MB patients and of clinical and bacteriological inactivity.

In the Addis Ababa area 218 TT, $685 \mathrm{BT}$ and $1005 \mathrm{BL}$ and LL patients were released from dapsone monotherapy during the period July 1983-July 1984.

Among the BT patients five relapses have been diagnosed; among the BL and LL patients, 57 relapses. The relapse rate in the BT patients is $7 \cdot 3 / 1000$ in about two years which is $3 \cdot 65 / 1000$ per year. The relapse rate in BL and LL patients is $57 / 1000$, which is $28 \cdot 5 / 1000$ per year. Data about the period between release from treatment and diagnosis of relapse is available on the patient record cards and will be analysed when our programme is computerized.

All relapsed patients presented themselves at our clinics. Active tracing of patients who do not come for regular examinations after release from treatment is not carried out. Therefore, it is not known whether these relapses represent only a proportion of the real number of relapses.

\section{Results of implementation of MDT}

\section{THE DEBRE BERHAN AREA}

In the Debre Berhan area 3509 patients were under chemotherapy by January 1983. Prior to the introduction of MDT only 26 PB patients were released from treatment (RFT). Due to incomplete recording of signs of inactivity it appeared not to be possible to apply guidelines defined by the WHO for release from treatment, ${ }^{4}$ while at the time of introduction of MDT instructions for RFT of patients in Ethiopia had not been defined. During the first months of 1983, 3140 patients were put under MDT. The remaining 369 patients continued dapsone monotherapy because they were either not able or willing to attend monthly for the supervised treatment or had attended very irregularly. Results of completion of treatment are evaluated through cohort analysis. The results of the four sixmonthly cohorts of PB patients for 1983 and 1984 are given in Table 1.

Of the 1944 PB patients who started MDT during 1983 and 19841715 patients $(88 \cdot 2 \%)$ completed their course of MDT and were released from treatment; 188 patients $(9 \cdot 7 \%)$ discontinued the treatment. Of the remaining 41 patients $(2 \cdot 1 \%)$ 12 died, 17 were transferred out and 12 continued the treatment after the 9 months which are allowed for completion of the course of MDT. These results are considered quite satisfactory. Compared with 1983 a decrease in the proportion 
Table 1. Results of completion of MDT of PB patients: Debre Berhan Area

\begin{tabular}{|c|c|c|c|c|c|c|c|c|c|c|}
\hline & \multicolumn{2}{|c|}{ Cohort 11983} & \multicolumn{2}{|c|}{ Cohort 21983} & \multicolumn{2}{|c|}{ Cohort 11984} & \multicolumn{2}{|c|}{ Cohort 21984} & \multicolumn{2}{|l|}{ Total } \\
\hline & No. patients & $(\%)$ & No. patients & $(\%)$ & No. patients & $(\%)$ & No. patients & $(\%)$ & No. patients & $(\%)$ \\
\hline Put under MDT & 1684 & - & 70 & - & 130 & - & 60 & - & 1944 & - \\
\hline Released from MDT & 1501 & $(89 \cdot 1)$ & 64 & $(91 \cdot 4)$ & 107 & $(82 \cdot 3)$ & 43 & $(7 \cdot 7)$ & 1715 & $(88 \cdot 2)$ \\
\hline MDT discontinued & 157 & $(9 \cdot 3)$ & 5 & $(7 \cdot 1)$ & 18 & $(13 \cdot 9)$ & 8 & $(13 \cdot 3)$ & 188 & $(9 \cdot 7)$ \\
\hline Died & 12 & $(0 \cdot 7)$ & - & - & - & - & - & - & 12 & $(0 \cdot 6)$ \\
\hline Transferred out & 14 & $(0 \cdot 8)$ & 1 & $(1 \cdot 4)$ & 1 & $(0 \cdot 7)$ & 1 & $(1 \cdot 7)$ & 17 & $(0 \cdot 9)$ \\
\hline Continued MDT & - & - & - & - & 4 & $(3 \cdot 1)$ & 8 & $(13 \cdot 3)$ & 12 & $(0 \cdot 6)$ \\
\hline
\end{tabular}


Table 2. MB patients struck off registers

\begin{tabular}{lc}
\hline & No. patients (\%) \\
\hline Released from MDT & $802(71 \cdot 3)$ \\
MDT discontinued & $120(10 \cdot 7)$ \\
Died & $68(6 \cdot 0)$ \\
Transferred out & $124(11 \cdot 0)$ \\
Left area without transfer & $10(0 \cdot 9)$ \\
Total & $1124(99 \cdot 9)$ \\
\hline
\end{tabular}

of patients who completed their course of MDT can be observed for the patients who started MDT during 1984, while the proportion of patients who discontinued the treatment increased. The severe drought situation which has affected large parts of the area since the end of 1984 has been identified as the main reason for these results.

The first cohort of MB patients for 1983 will be evaluated by September 1986.

During the period January 1983-January 19861124 MB patients have been struck off the registers, for reasons given in Table 2. The majority of the 120 patients who discontinued the treatment had attended regularly during 1983 and 1984 , had received dapsone prior to MDT and had negative skin smears before the start of MDT or after 1 year of MDT. Therefore many of them may be considered to have received sufficient chemotherapy; however, as they were not formally released from treatment after clinical and bacteriological assessment, they are considered not to have fulfilled our criteria for release from treatment. ${ }^{3}$ The main reason for discontinuation of the treatment has been the severe drought situation. This also applies to the high number of deaths. Due to resettlement of large groups of the population the number of patients who were transferred is high.

Until January 1986 one BT relapse has been diagnosed in a patient who did not receive dapsone prior to MDT.

By January 19862268 patients were reported to be under chemotherapy, 1877 patients under MDT and 391 patients under dapsone monotherapy. In this area the number of patients under chemotherapy has been reduced to $56 \%$ within a period of $2 \frac{1}{2}$ years.

THE ADDIS ABABA AREA

In the Addis Ababa area 1952 patients were released from chemotherapy prior to introduction of MDT. From March 1984 to January 19862744 patients, 1861 MB and 883 PB patients, have been put under MDT. Results of completion of treatment of the two six-monthly cohorts of PB patients for 1984 are given in 
Table 3. Of the 494 patients who started MDT during 1984484 (98.0\%) completed their course of treatment; a very satisfactory result. By January 1986 no MB patients had been released from MDT. Over $90 \%$ of the MB patients attended regularly for their treatment.

Compliance studies, by way of the urine spot test for the presence of dapsone, are carried out with the PB patients during the 4th and 6th supervised treatment round and with the $\mathrm{MB}$ patients during the 4th, 6th, 12th, 18th and 24th treatment round. The results for the fourth up to the 18 th treatment round are given in Table 4. The results are encouraging, especially when compared with results of previous compliance studies carried out at ALERT, which gave 60-70\% positive urines.

The decline in proportion of positive urines, especially during the eighteenth treatment round, could indicate a decline in motivation of patients for the new treatment.

By January 19862268 patients were reported to be under chemotherapy, 1877 patients under MDT and 391 patients under dapsone monotherapy. In this

Table 3. Results of completion of treatment of PB patients: Addis Ababa Area

\begin{tabular}{|c|c|c|c|c|c|c|}
\hline \multirow[b]{3}{*}{ Put under MDT } & \multirow{2}{*}{\multicolumn{2}{|c|}{$\begin{array}{c}\text { Cohort } 11984 \\
\text { No. patients (\%) }\end{array}$}} & \multirow{2}{*}{\multicolumn{2}{|c|}{$\begin{array}{c}\text { Cohort } 21984 \\
\text { No. patients (\%) }\end{array}$}} & \multicolumn{2}{|c|}{ Total } \\
\hline & & & & & \multicolumn{2}{|c|}{ No. patients $(\%)$} \\
\hline & 386 & - & 108 & - & 494 & - \\
\hline Released from MDT & 382 & $(99 \cdot 0)$ & 102 & $(94 \cdot 4)$ & 484 & $(98 \cdot 0)$ \\
\hline MDT discontinued & 3 & $(0 \cdot 8)$ & 5 & $(4 \cdot 6)$ & 8 & $(1 \cdot 6)$ \\
\hline Died & - & - & - & - & - & - \\
\hline Transferred out & 1 & $(0 \cdot 2)$ & 1 & $(0 \cdot 9)$ & 2 & $(0 \cdot 4)$ \\
\hline Continued MDT & - & - & - & - & - & - \\
\hline
\end{tabular}

Table 4. Results of urine spot test during different treatment rounds

\begin{tabular}{lrrr}
\hline & & & \\
& Urines tested & positive $(\%)$ & negative $(\%)$ \\
\hline 4th round & 1311 & $1194(91 \cdot 0)$ & $117(8 \cdot 9)$ \\
6th round & 1284 & $1101(85 \cdot 8)$ & $183(14 \cdot 3)$ \\
12th round & 927 & $795(85 \cdot 8)$ & $132(14 \cdot 2)$ \\
18th round & 1002 & $768(76 \cdot 6)$ & $234(23 \cdot 4)$ \\
Total & 4524 & $3858(85 \cdot 3)$ & $666(14 \cdot 7)$ \\
\hline
\end{tabular}


area the number of patients under chemotherapy has been reduced to $56 \%$ within a period of $2 \frac{1}{2}$ years.

\section{THE SOUTHERN SHOA AREA}

In the Southern Shoa area 2104 patients were released from treatment prior to introduction of MDT.

In the two districts the urine spot test for the presence of dapsone was carried out on a sample of patients who had been selected for MDT, prior to their start of MDT. Out of the 649 urines which were tested 438 were positive $(67 \cdot 5 \%)$. The result is similar to that obtained at ALERT during the dapsone monotherapy era. Compliance data on the self-administration of dapsone before implementation of MDT will be compared with those after MDT has been introduced.

By January 1986, one month after the start of implementation of MDT, 1190 patients, $732 \mathrm{MB}$ and $458 \mathrm{~PB}$ patients, had started their course of MDT, while 1157 patients were still under dapsone monotherapy.

\section{Prospects for leprosy control in the ALERT Leprosy Control Programme}

During the period July 1982-July 1985 the number of patients under chemotherapy, MDT and dapsone monotherapy, in Shoa Region, decreased from 20,908 to 10,507 ; a reduction of $50 \%$ within a period of 3 years. This decrease is due to the application of instructions for release from treatment of patients after monotherapy ${ }^{3}$ and the introduction of MDT.

By 1993, 3 years after the last district of Shoa Region will have been included in the MDT programme, all but a few of the patients under chemotherapy, will be newly diagnosed patients and patients who have relapsed, who hopefully will be few.

Assuming that there will not be major changes in the number of newly diagnosed patients in the region, the number of patients under chemotherapy will, from 1993 onwards, be in the order of $2000-3000$ at any time. This is a reduction of $85-90 \%$ in the number of patients under antileprosy treatment within a period of 10 years. With 292 leprosy diagnostic and treatment centres in the region, the average number of patients per clinic will then be from seven to ten. If the decline in detection of new patients, which has been observed during the last few years, continues, the number of patients under chemotherapy will be even less. Although the workload, related to the number of patients under chemotherapy, will gradually decrease, the total workload will continue to remain high for many years due to:

1 Provision of care directed at existing and potential increase of disabilities. In the ALERT Leprosy Control Programme about 35\% of the known leprosy 
patients are in need of regular care for exıstıng disabilities or threatening increased disability. If the trend in steady, although slow, decrease in the proportion of new patients who have already severe disabilities at the time of diagnosis, a trend which has been observed during the last few years, continues, and if reactions are diagnosed early and properly treated and continuous care can be given to prevention of increase of disability, this group of patients will gradually decrease. However this decrease is not expected to be very fast. The figure for 1985 of $18 \%$ of new patients who had already a disability grade 2 or 3 at the time of diagnosis of leprosy is still high. Our method of case-detection has almost exclusively been passive over the years. A more active detection of patients, an approach which has been formulated as one of our priorities, whenever feasible, could lead to a steeper decline in the proportion of new patients with severe disability.

2 The need for regular follow-up examinations of patients who have been released from MDT. So far about $40 \%$ of the patients have attended for the appointed follow-up examinations. With a follow-up period of 5 years after release from treatment, the group of patients who need to come for follow-up examinations will gradually decrease. In the ALERT Leprosy Control Programme this group will, from 1998 onwards, comprise mainly newly diagnosed patients.

3 Another matter for serious concern is the integration 'of leprosy and tuberculosis control activities. The leprosy control infrastructure could provide a sound basis for extension of tuberculosis control in the rural areas $(1 \cdot 5)$.

\section{MDT field evaluation studies}

It is planned to carry out three MDT field evaluation studies in 41 out of the 48 clinics of the Addis Ababa MDT area.

These studies concern: frequency of occurrence of relapses after treatment; frequency of occurrence of leprosy reactions during and after treatment; and nerve function impairment during and after treatment.

The leprosy control activities in the 41 study clinics will remain basically the same as in all other clinics where MDT has been implemented in the ALERT Leprosy Control Programme.

However, there are some differences:

1 For MB patients the duration of the MDT course is limited to 2 years, while in the routine services the treatment is continued till skin smear (Bacteriological Index) negativity.

2 The surveillance of patients after the chemotherapy course is 'active' in the study clinics, while 'passive' in the routine service clinics.

3 The study clinics will generally have more intensive supervision and for 
quality control some additional assessments are done, e.g. urine spot test for dapsone intake compliance.

\section{Problems encountered with implementation of MDT}

The following problems were encountered during the implementation of MDT:

\section{The severe drought situation}

Large parts of the Debre Berhan area were affected by the severe drought. This has caused a decline in attendance by MB patients and by a high number of patients who were, because of resettlement, transferred to a non-MDT area.

\section{Acute shortages of public transport}

Because many of the public buses were used in resettlement programmes, we experienced acute shortages of public transport. Field staff had to rely on alternative ways of transportation, and often they had to walk to the clinics.

\section{Analysis of data}

The amount of paperwork has substantially increased. Compilation of analysis 'by hand' of the many data which are essential for evaluation of MDT and the leprosy situation over the years have become very time consuming tasks. At present much of the available data remains unused.

\section{Differentiation between relapse and reversal reaction after release from treatment}

So far there are no criteria for distinction between relapse and reversal reaction af ter release from treatment. During the first months of 1986 six BT patients from the Debre Berhan MDT area were diagnosed with a reactivation of the disease with involvement of nerves, 12 months or more after their release from MDT.

\section{References}

1 Becx-Bleumink, M. Annual Report of ALERT for 1985, 72-110.

2 World Health Organization. Chemotherapy of leprosy for control programmes. Report of a WHO Study Group. Technical Report Series 675; 1982.

3 All African Leprosy and Rehabilitation Training Centre. Manual for implementation of Multiple Drug Therapy, 1985.

${ }^{4}$ World Health Organization. A guide to leprosy control, 1980; 27-28.

${ }_{5}$ Becx-Bleumink, M. New developments in ALERT Leprosy Control Programme and the issues of integration. Eth J of Health Development 1984; 2: 49-55. 